\title{
Disseminated intravascular coagulation: enumerating studies of Lemierre's syndrome and Covid19 to highlight the similarities between these diseases Sandeep Chakraborty
}

\section{Letter}

Disseminated intravascular coagulation (DIC) is a serious disorder characterized by small blood clots developing throughout the bloodstream, blocking small blood vessels, depleting platelets and clotting factors leading to excessive bleeding. Lately, it is been reported that Covid19 is more of a vascular problem, than one of the lungs. Here, I enumerate studies of Lemierre's syndrome with DIC, and also papers highlighting the Covid19 cases - this is based on my hypothesis that Covid19 is very similar to Lemierre's Syndrome, wherein an anaerobic bacteria is enabled by a virus (SARS-Cov2/EBV) to form an abscess, from which a blood clot (carrying the bacteria) disseminates through the blood stream [1].

\section{Lemierre's Syndrome and DIC}

1. A 1988 paper ' 23 year old woman died within six hours of admission from acute DIC. Fusobacterium necrophorum was isolated from the blood' [2].

2. A 1990 paper reported a previously healthy 42-year-old man had a severe Fusobacterium necrophorum septicemia with DIC [3]. 'Peripheral, painful, cyanotic and gangrenous lesions appeared on toes, external ears and nose tip' and warned that the 'growth of these bacteria in blood cultures is slow and confirmation of the infection may thus be delayed' [3].

3. In 1990-95 in Denmark, it was reported that 'disseminated intravascular coagulation was mild and not associated with increased mortality' [4].

4. In 2007, a patient with Lemierre's Syndrome reported 'decreased platelet count and increased D-dimers the patient appeared to be in early sepsis and disseminated intravascular coagulation' [5]. Complete recovery.

5. In 2012 in India, 'a healthy toddler who presented with acute sore throat that led to bacteremia, septic shock, disseminated intravascular coagulopathy, encephalopathy and pleural effusion' [6]. 'He was successfully treated with antibiotics and low molecular weight heparin.'

6. In 2017 another case of Lemierre's syndrome showed 'severe inflammatory response, respiratory failure, septic shock, acute kidney injury and mild disseminated intravascular coagulation was attributed to an atypical pneumonia' [7].

\section{Covid19 and DIC}

ISTH has issued an interim guidance for recognition and management of coagulopathy, specifically to monitor coagulation markers [8].

1. On March 13 Tang, et at noted ' $71.4 \%$ of non-survivors and $0.6 \%$ survivors met the criteria of disseminated intravascular coagulation during their hospital stay" in 183 patients [9].

2. On April 9, venous thromboembolism(VTE) incidence of VTE in 81 severe patients was 25\%(20/81), of which 8 patients died. The D-dimer in severe NCP patients was reported to be a good index for identifying high-risk groups of VTE [10].

3. On April 18, Kollias, et. al noted the risks of 'coagulopathy namely disseminated intravascular coagulation, which has a rather pro-thrombotic character with high risk of venous thromboembolism' [11]. 
4. On 28th April, in a contrarian statement to most Covid19 study, Leisman et al. noted that 'there is no evidence that COVID-19 patients develop cytokine storm', and the use of cytokine-blockade agents does not have evidence of benefit [12]

5. On April 30, Lang, et. al suggest alveolar damage is not key in hypoxaemia in COVID-19 pneumonia (doi.org/10.1016/S1473-3099(20)30367-4) They observed 'a peripheral halo of increased perfusion has not been described in pulmonary infarction, but has been described once previously in a case of bacterial pneumonia'. Once again, they used culturing to determine bacterial infection, which is not a sensitive method to detect secondary infection. Also, a 2016 study reported 'ground-glass opacities (halo sign), findings suggestive of invasive pulmonary aspergillosis', a fungal infection [13].

\section{These anaerobes are difficult to culture - so dont depend on culturing}

I cite this case report with fatal CNS complications in Lemierre's Syndrome - 'Microbial identification can take several days due to the slow growing nature. One case report describes the culture taking 9 days to grow while another took 2 weeks for isolation [5]. Unfortunately, no organism was ever isolated in our case.' [?]. And 'growth of these bacteria in blood cultures is slow and confirmation of the infection may thus be delayed' [3].

\section{References}

1. Chakraborty S (2020). Sars-cov2 enables anaerobic bacteria (prevotella, et al) to colonize the lungs disrupting homeostasis - symptoms (ards, septic shock, blood clots, arterial stroke) finds resonance, with key differences, in the 'forgotten disease' lemierre syndrome, caused by anaerobic bacteria enabled by epstein barr virus. doi:10.31219/osf.io/usztn. URL osf.io/usztn.

2. Potter M, Drysdale H, Price P, Buck A (1988) Disseminated intravascular coagulation with fusobacterium necrophorum septicaemia. Postgraduate medical journal 64: 155-156.

3. Page Y, Comtet C, Tardy B, Zeni F, Thevenet D, et al. (1990) Disseminated intravascular coagulation in fusobacterium necrophorum septicemia. Scandinavian journal of infectious diseases 22: 743-747.

4. Hagelskjaer L, Prag J, Malczynski J, Kristensen J (1998) Incidence and clinical epidemiology of necrobacillosis, including lemierre's syndrome, in denmark 1990-1995. European journal of clinical microbiology and infectious diseases 17: 561-565.

5. Hlibczuk V (2007) Lemierre's syndrome complicating bacterial pharyngitis in a patient with undiagnosed factor xii deficiency. The Journal of emergency medicine 32: 365-369.

6. Khan A, Ganesan S, Arora M, Hussain N (2013) Life threatening complication of sore throat: Lemierre's syndrome. The Indian Journal of Pediatrics 80: 1059-1061.

7. Rae J, Misselbrook K (2017) Lemierre's syndrome-a rare cause of disseminated sepsis requiring multiorgan support. Journal of the Intensive Care Society 18: 329-333.

8. Thachil J, Tang N, Gando S, Falanga A, Cattaneo M, et al. (2020) Isth interim guidance on recognition and management of coagulopathy in covid-19. Journal of Thrombosis and Haemostasis .

9. Tang N, Li D, Wang X, Sun Z (2020) Abnormal coagulation parameters are associated with poor prognosis in patients with novel coronavirus pneumonia. Journal of Thrombosis and Haemostasis .

10. Cui S, Chen S, Li X, Liu S, Wang F (2020) Prevalence of venous thromboembolism in patients with severe novel coronavirus pneumonia. Journal of Thrombosis and Haemostasis . 
11. Kollias A, Kyriakoulis KG, Dimakakos E, Poulakou G, Stergiou GS, et al. (2020) Thromboembolic risk and anticoagulant therapy in covid-19 patients: Emerging evidence and call for action. British Journal of Haematology .

12. Leisman DE, Deutschman CS, Legrand M Facing covid-19 in the icu: vascular dysfunction, thrombosis, and dysregulated inflammation. Intensive Care Medicine : 1.

13. Choi MH, Jung JI, Chung WD, Kim YJ, Lee SE, et al. (2014) Acute pulmonary complications in patients with hematologic malignancies. Radiographics 34: 1755-1768. 